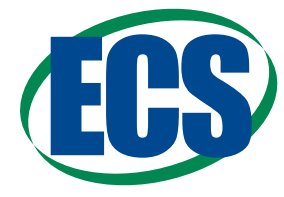

JSS Focus Issue on Thermoelectric Materials \& Devices

\title{
Review-Organic Materials for Thermoelectric Energy Generation
}

\author{
Lewis M. Cowen, ${ }^{\text {a Jonathan Atoyo, }},{ }^{\text {b Matthew J. Carnie, }},{ }^{\text {b,z }}$ Derya Baran,,${ }^{c, z}$ \\ and Bob C. Schroeder ${ }^{a, z}$
}

${ }^{a}$ Organic Thermoelectrics Laboratory, Materials Research Institute and School of Biological \& Chemical Sciences,
Queen Mary University London, London E1 4NS, United Kingdom
${ }^{b}$ SPECIFIC IKC \& Materials Research Centre, College of Engineering, Swansea University, Bay Campus, Swansea
SA1 8EN, United Kingdom
${ }^{c}$ Physical Sciences and Engineering Division, KAUST Solar Center (KSC), King Abdullah University of Science and
Technology (KAUST), Thuwal, 23955-6900, Kingdom of Saudi Arabia

Organic semiconductor materials have been promising alternatives to their inorganic counterparts in several electronic applications such as solar cells, light emitting diodes, field effect transistors as well as thermoelectric generators. Their low cost, light weight and flexibility make them appealing in future applications such as foldable electronics and wearable circuits using printing techniques. In this report, we present a mini-review on the organic materials that have been used for thermoelectric energy generation.

(C) The Author(s) 2017. Published by ECS. This is an open access article distributed under the terms of the Creative Commons Attribution 4.0 License (CC BY, http://creativecommons.org/licenses/by/4.0/), which permits unrestricted reuse of the work in any medium, provided the original work is properly cited. [DOI: 10.1149/2.0121703jss] All rights reserved.

(c) BY

Manuscript submitted November 15, 2016; revised manuscript received January 12, 2017. Published January 28, 2017. This paper is part of the JSS Focus Issue on Thermoelectric Materials \& Devices: Phonon Engineering, Advanced Materials and Thermal Transport.

The reduction in the effects of climate change, caused by the burning of fossil fuels, has recently been given increased emphasis by major world governments and it is therefore necessary to consider the efficiency of the methods we currently use to generate energy. It has previously been estimated that $63 \%$ of global energy consumption is wasted with the major form of this waste being heat. ${ }^{1,2}$ It is therefore important to develop methods of reconverting this wasted heat back in to useful forms of energy, such as electricity.

Thermoelectric generators (TEG) are a promising technology which make use of a process known as the Seebeck effect for heat recovery. The Seebeck effect can be described as two dissimilar conductors or semiconductors connected thermally in parallel and electrically in series and exposed to a temperature gradient causing a difference in voltage between the two materials. ${ }^{3,4}$ In a TEG one p-type and one ntype semiconductor make use of the Seebeck effect by connecting one to the other electrically in series and thermally in parallel; a general device setup is shown in Figure 1. By connecting the two components, by a junction which is heated, the n-type component of the device transports electrons from the hot junction to a heat sink while the p-type material transports positively charged holes along the same direction of the temperature gradient. The reverse is also possible if the holes and electrons flow away from a cold connecting junction to a heated point at the end of each semiconductor, this induces a constant cooling at the junction through the Peltier effect. ${ }^{4,5}$

The quantification of the Seebeck effect, in a specific material, is given by the Seebeck coefficient, $\alpha$, and is the open circuit voltage of a material subjected to a temperature gradient, commonly with units on the scale of $\mu \mathrm{VK}^{-1}$ to $\mathrm{mVK}^{-1}{ }^{6} \mathrm{~A}$ materials overall efficiency in the conversion of heat to electricity is given by the dimensionless figure of merit, ZT, defined as,

$$
Z T=\frac{\alpha^{2} \sigma T}{\kappa}
$$

where $\sigma$ is the electrical conductivity and $\kappa$ is the thermal conductivity. ${ }^{6-8}$ It is therefore necessary for thermoelectric materials to have a high electrical conductivity and a low thermal conductivity making electrically conducting metals inappropriate for thermoelectric applications due to their high thermal conductivities and low

${ }^{\text {z} E-m a i l: m . j . c a r n i e @ s w a n s e a . a c . u k ; ~ d e r y a . b a r a n @ k a u s t . e d u . s a ; ~ b . c . s c h r o e d e r @ q m u l ~}$ .ac.uk
Seebeck coefficients. The ideal thermoelectric materials should be an electrical crystal to maximize $\sigma$ and at the same time a phonon glass to minimize $\kappa$. Generally a material that is a good electrical conductor is also good at conducting heat, since both charge carriers and phonons carry heat. ${ }^{9}$ Also this inverse relationship between Seebeck coefficient and electrical conductivity complicates the design of organic semiconductors for thermoelectric applications and optimizing one condition quite often has a detrimental effect on the other. In case thermal data for a given material is not available, the thermoelectric quality of a material can be described by the power factor (PF) given by the equation,

$$
P F=\alpha^{2} \sigma
$$

The nature of the semiconducting materials used is dependent on the nature of the waste heat which is being supplied to the TEG. The use of doped inorganic semiconductors as thermoelectric materials, usually based on the semimetals silicon and germanium, has been widely explored for the recovery of waste heat at temperatures above $250^{\circ} \mathrm{C}^{8}$ Approximately $50 \%$ of waste heat is stored at temperatures of less than $250^{\circ} \mathrm{C}$ and large areas of processed thermoelectric material

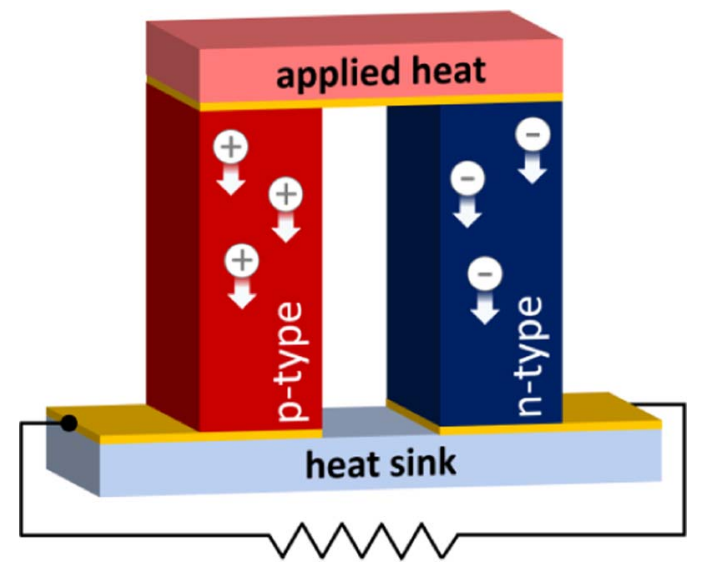

Figure 1. Schematic representation of a thermoelectric generator (TEG), showing both the hole transporting p-type and the electron transporting $n$ type leg, electrodes are shown in yellow. 
are needed to recover this energy, therefore far fewer thermoelectric materials capable of efficiently recovering this energy have been developed. ${ }^{6}$ Unlike the materials used for inorganic thermoelectric devices, organic thermoelectric materials are made from abundant and less toxic elements. In addition, organic materials can be processed from solution, making it possible to deposit the materials over large areas using cheap printing techniques, such as inkjet printing, rollto-roll printing, gravure printing, etc., leading to economically viable TEG, more suited to efficiently recovering heat at low operating temperatures. It is noteworthy that organic materials generally have lower electrical conductivities than inorganic semiconductors, and although the lower thermal conductivities do not compensate for this enough when comparing thermoelectric efficiencies of the organic materials to inorganics, the advantages described above for ease of processing and abundancies of materials make organic semiconductors worth studying for thermoelectric applications.

\section{Doping in Organic Semiconductors}

The mechanisms by which organic semiconductors conduct electrons or holes cannot be easily described in the same way that inorganic semiconductors have been, which is through band theory. According to band theory, the highest occupied energy levels of an atom in a lattice form a valence band and the lowest unoccupied electronic levels form the conduction band; electrical conductivity in semiconductors arises when the bandgap between these two bands is small enough for an electron to move from the valence band to the conduction band under excitation. Through the introduction of defects, by doping, additional energy levels can be introduced between the valence and conduction bands reducing the bandgap. Insulators have a wide bandgap which cannot be crossed whereas full conductors do not possess a bandgap and the valence and conduction bands overlap. ${ }^{10,11}$

Over the last two decades, many examples of organic conducting polymers have been demonstrated, however most require an external species, a dopant, to increase conductivities from regimes as low as $10^{-8} \mathrm{~S} \mathrm{~cm}^{-1}$ in undoped polymers to as high as $10^{4} \mathrm{~S} \mathrm{~cm}^{-1}$ in doped semiconductors. By controlling the concentration of dopants in a material it is possible to tune the conductivity of a polymer. Unlike inorganic semiconductors, in which an impurity is introduced to a crystalline semiconductor to reduce the gap between the valence and conduction bands, doping in organic semiconductors requires the addition of strongly electron donating (n-type) or strongly electron withdrawing (p-type) molecular species which induce mobile charge carriers along the polymer backbone. ${ }^{10,12}$ A p-type dopant can be thought of as an oxidizing agent which removes an electron from the highest occupied molecular orbital (HOMO) of the organic semiconductor, introducing a positive hole along the backbone. An n-type dopant on the other hand, is a reducing agent which donates an electron to the lowest unoccupied molecular orbital (LUMO) of the organic semiconductor introducing a negative charge carrier. ${ }^{12}$ The integer charge transfer model and the molecular orbital levels involved in the doping processes (p-type and n-type) are schematically represented in Figure 2.

More recently it has emerged however that the integer charge transfer model is a simplified model to describe the doping in organic semiconductors. A more accurate description of organic semiconductor doping is described in the hybrid charge transfer model. In contrast to the discrete energy levels involved in the integer charge transfer model, the hybrid charge transfer model describes the doping of organic semiconductors via the formation of charge transfer states arising from orbital mixing between donor and acceptor orbitals. ${ }^{13}$ For a more comprehensive discussion on the hybrid charge transfer
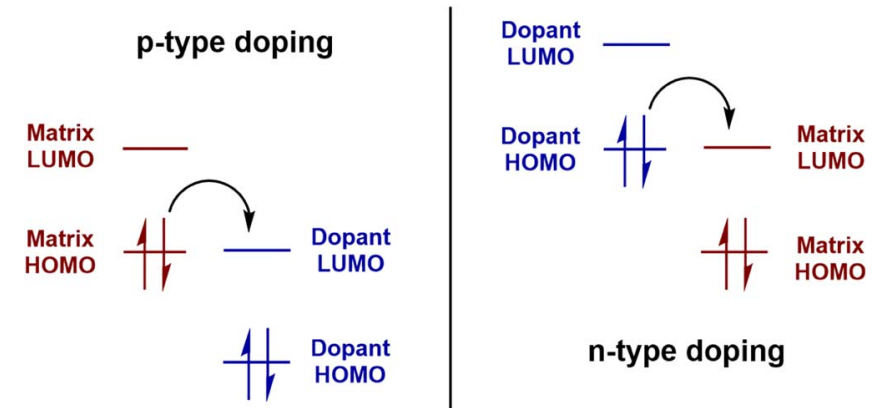

Figure 2. Organic semiconductor doping via integer charge transfer model.

model and doping in organic semiconductors, the reader is referred to another review if required. ${ }^{14}$

Some of the earliest examples of conducting organic materials come from the p-type doping of polyacetylene with halogens. ${ }^{15,16} \mathrm{In}$ these early conducting polymers, dopants were introduced via exposure to halogen vapors leading to diffusion problems during processing. A more common and practical approach is to use small molecules as dopants. P-type doping has been widely explored with strong Lewis acids being employed. ${ }^{17,18} \mathrm{~N}$-doping of materials is much more difficult to achieve since the HOMO of the dopant must be high to allow it to donate electrons to the LUMO of various materials, however having such a high-energy HOMO makes the dopant more resistive to the oxidation it is required to undergo. ${ }^{12} \mathrm{~N}$-doping has previously been achieved using alkali metals, mostly lithium, ${ }^{19-21}$ and molecules with extremely high HOMO levels such as tetrathianaphthacene $\left(\mathrm{TTN}, \mathrm{E}_{\mathrm{HOMO}}=-4.7 \mathrm{eV}\right.$ ), which doped hexadecafluoro-zincphthalocyanine (F16ZnPc) with a LUMO energy level of $-4.5 \mathrm{eV}$. The same dopant however did not dope tris(8-hydroxyquinoline)aluminum (Alq3) with a LUMO energy level of $-2.5 \mathrm{eV}$, showing that similar energies in the HOMO of the dopant and LUMO of the acceptor are essential. ${ }^{22}$

In p-type doping the polymer is oxidized by the dopant this produces a positive cation and a free radical electron on the polymer, the charge neutrality is maintained by the formation of an anion on the reduced dopant. If this process occurs twice in trans-polyacetylene for example (Figure 3), the two radical electrons can recombine to form what is known as a spinless soliton, a positive or negative charge carrier free to independently move around the polymer chain. The process of forming solitons is unique to trans-polyacetylene due to it containing a doubly degenerate ground state which allows the soliton charges to move independently of each other. ${ }^{23-26}$

Most conjugated polymers however do not exhibit the same symmetry as trans-polyacetylene and therefore do not contain the degenerate ground state necessary for independent solitons to act as charge carriers. Considering p-type doping, an electron is removed from the polymer and the cation and radical are again formed; the resultant cation and radical electron are partially delocalized over the $\pi$-conjugated system and is termed a polaron (Figure 4). The polaron induces the formation of the quinoidal structure leading to a distortion in bond lengths over the section of the polymer linking the radical and cation. This distortion is not energetically favored and is therefore usually stabilized over more than one monomer unit. Upon further oxidation, which can be induced by increasing dopant concentration, the unpaired radical electron of the polaron can be removed which leads to a sequence of quinoid type monomer units along the polymer which are separated from the normal aromatic monomer units by the two cationic charges. These sections of the polymer are known

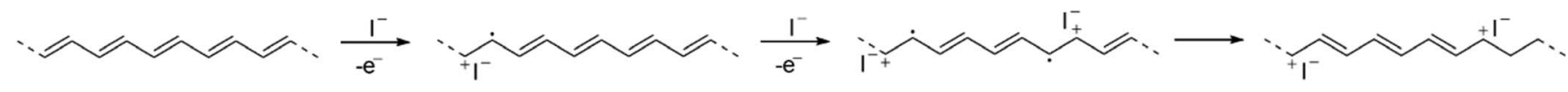

Figure 3. Soliton formation using iodine as the dopant trans-polyacetylene. 


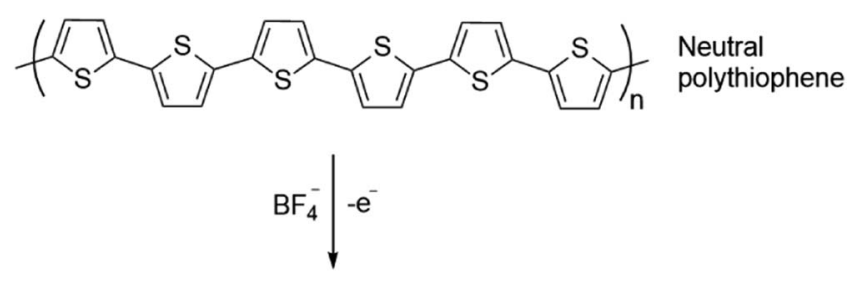

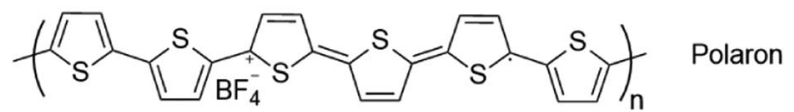
$\mathrm{BF}_{4}^{-} \downarrow-\mathrm{e}^{-}$

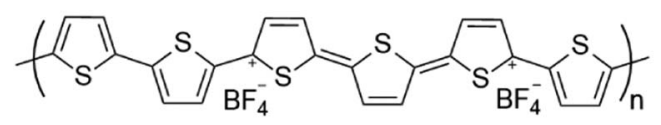

Bipolaron

Figure 4. Bipolaron formation in polythiophene upon doping with $\mathrm{BF}_{4}{ }^{-}$.

as bipolarons and at high dopant levels are responsible for charge transport across the polymer.

\section{P-type Organic Thermoelectric Materials}

In organic thermoelectric research, efforts in searching for thermoelectric materials have mostly focused on conjugated polymers and charge transfer complexes which are p-type. The first conducting organic polymer to be extensively studied was transpolyacetylene. ${ }^{15,16,24-26}$ Conductivities of $560 \mathrm{~S} . \mathrm{cm}^{-1}$ and high carrier mobilities of approximately $1 \mathrm{~cm}^{2} \mathrm{~V}^{-1} \mathrm{~s}^{-1}$, due to polyacetylene linearity, were reported when $\mathrm{p}$-doped with arsenic pentafluoride $\left(\mathrm{AsF}_{5}\right)$. When doped with iodine vapor the electrical conductivity could be further increased to $10,000 \mathrm{~S} . \mathrm{cm}^{-1} \cdot{ }^{27}$ Roth et al. were the first to measure the thermoelectric properties of polyacetylene doped with transition metal halides. ${ }^{28}$ Electrical conductivities of up to $30,000 \mathrm{~S} . \mathrm{cm}^{-1}$ were measured in oriented polyacetylene samples at $220 \mathrm{~K}$, with power factors (PF) as high as $2 \times 10^{-4} \mathrm{~S} . \mathrm{cm}^{-1} .^{29}$ Despite those promising results, polyacetylene is unsuitable for thermoelectric applications, mainly due to its poor solubility and oxidative stability. Nevertheless, those initial results on polyacetylene sparked the interest in organic thermoelectric materials, particularly into organic p-type materials which are more stable under atmospheric processing and operating conditions.

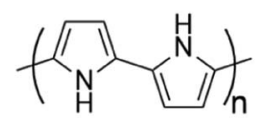

polypyrrole

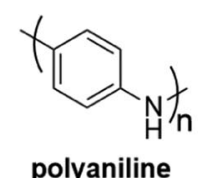

polyaniline

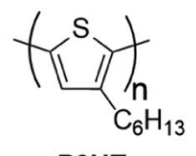

P3HT

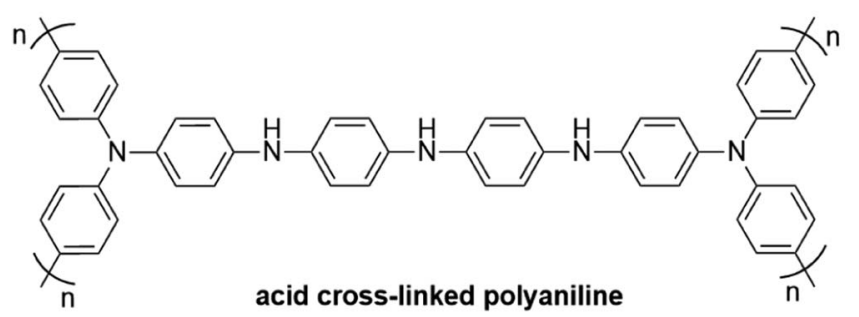

Figure 5. Chemical structures of several popular p-type organic semiconductors.
Due to its facile synthesis, good solubility and high intrinsic conductivity, conducting polypyrroles have been extensively studied over the years. The polymer can be synthesized either via oxidative polymerization using pyrrole monomers by iron(III) trichloride $\left(\mathrm{FeCl}_{3}\right)^{30}$ or electrochemically. The electrochemical synthesis however is often preferred because it allows to systematically introduce dopant anions, such as ferrocyanide $\left(\mathrm{Fe}(\mathrm{CN})_{6}{ }^{3-}\right)^{31}$ during the synthesis or alternatively during the formation of thin films. ${ }^{32}$ Conductivities in polypyr-

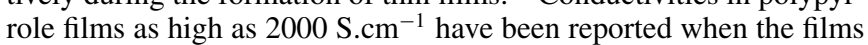
were synthesized by freezing interfacial polymerization. ${ }^{33}$ The high conductivities are thought to be due to the specific synthetic method used, causing an increased order in the polymeric structure allowing for greater overlap of the molecular orbitals. Depending on the dopant and synthetic method used, the Seebeck coefficient in polypyrroles exceeds $10 \mu \mathrm{V} . \mathrm{K}^{-1}$ at $250 \mathrm{~K} \cdot{ }^{34}$

Like polypyrrole, polyaniline (PANI) is yet another very popular conducting polymer because of its versatile and easy synthesis and its good stability (See Figure 6). The synthesis can be carried out chemically or electrochemically, leading to materials with differing electrical conductvities. ${ }^{35}$ It was found that electrochemical synthesis lead to lower conductivities of $1.7 \times 10^{-4} \mathrm{~S} . \mathrm{cm}^{-1}$ compared to 6.3 $\times 10^{-4}{\mathrm{~S} . \mathrm{cm}^{-1}}$ for the chemically synthesized polymer., accompanied by the formation of less bipolaron charge carriers and a lower crystallinity. Studies have shown that varying factors such as type of dopant and concentration of dopant can lead to increased conductivity, compared to the neutral polymer. ${ }^{36,37}$ Interestingly it has been reported that the cross-linking of monomer repeat units in polyaniline derivatives can have an effect on the charge transport between chains and increased mobility and conductivity has been observed due to an increase in order between the polymer chains. ${ }^{38}$ An increase in conductivity of $25 \%$ was observed when compared to the linear polymer and was attributed to easier transport of charge between polymer chains; $\mathrm{H}_{2} \mathrm{SO}_{4}$ was used as the dopant.

$\mathrm{Li}$ et al. studied the dependence of the doping concentrations $(\mathrm{HCl})$ on the thermoelectric properties of PANI. At elevated doping levels the PANI shows metallic temperature dependence and maximum conductivities of $601{\mathrm{~S} . \mathrm{cm}^{-1}}$ at $303 \mathrm{~K}$ are reached. ${ }^{39}$ In line with the observed metallic behavior, the Seebeck coefficient increases linearly with increasing temperature. The maximum ZT value of $2.7 \times 10^{-4}$ for $\mathrm{HCl}$ doped PANI was measured at $423 \mathrm{~K}$.

Poly(3-hexylthiophene) (P3HT) is an alkylated derivative of polythiophene and its structure is shown in Figure $5 .^{40} \mathrm{P} 3 \mathrm{HT}$ is one of the most studied conjugated polymers, because of its commercial availability, excellent solubility and film forming properties. Therefore, it comes to no surprise that after P3HT has been very successfully introduced into organic photovoltaics and organic field effect transistors, researchers are interested in exploiting the polymers properties for organic thermoelectric applications. When electrostatically doped,

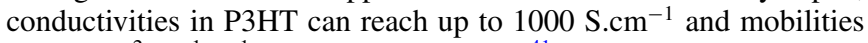
of $0.7 \mathrm{~cm}^{2} . \mathrm{V}^{-1} \cdot \mathrm{s}^{-1}$ have been measured. ${ }^{41}$ Electrostatic doping involves the introduction of charges through a gate, rather than through chemical oxidation, and is the method of doping used in organic fieldeffect transistors (OFET). The electrostatic charge injection allows P3HT to exhibit a high concentration of charge carriers, the density of p-type holes was estimated to be $10^{15} \mathrm{~cm}^{-2}$, which is above the value of $10^{13} \mathrm{~cm}^{-2}$ measured for silicon oxide $\left(\mathrm{SiO}_{2}\right)$ typically used as an insulator in OFET. Xuan et al. were interested in evaluating the thermoelectric properties of regio-regular P3HT using nitrosonium hexafluorophosphate $\left(\mathrm{NOPF}_{6}\right)$ as dopant. At low doping levels the large $\mathrm{PF}_{6}{ }^{-}$anions prevent an ordered structure within the $\mathrm{P} 3 \mathrm{HT}$, but with increasing doping levels the disorder within the polymer matrix diminishes and so does the Seebeck coefficient, whilst the electrical conductivity increases. ${ }^{42}$ The maximum power factor was obtained at doping levels ranging from 20 to $31 \%$, and the results showed that in conjugated polymers the thermoelectric properties depend on more than simply doping levels and that molecular order plays a key role for charge transport in doped organic semiconductors. Recently Zhu et al. reported power factors of $20 \mu \mathrm{W} \cdot \mathrm{m}^{-1} \cdot \mathrm{K}^{-1}$ in P3HT doped by the ferric salt TFSI $^{-} .43$ 


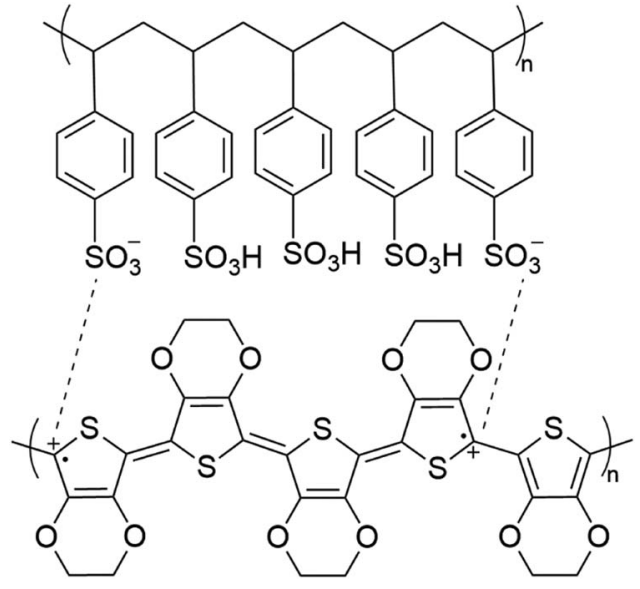

Figure 6. Chemical structures of (poly(3,4-ethylenedioxythiophene) (PEDOT) and poly(styrenesulfonate) (PSS).

Recent work by Sirringhaus and colleagues has concentrated on the measurement of Seebeck effects in poly(2-5-bis(3-alkylthiophen2-yl)thieno[3,2-b]thiophene) (pBTTT) and P3HT. ${ }^{61,62}$ Sirringhaus and Zhu have recently investigated how the Seebeck coefficient in p-type pBTTT and P3HT varies in organic field effect transistors upon differentiating factors such as gate voltage. ${ }^{63}$ This study was carried out as an alternative to measuring thermoelectric properties in chemically doped organic semiconductors due to the morphological changes that can be seen in these materials when doped.

The most commonly used and developed p-type material for thermoelectric applications is PEDOT:PSS, a polymer mixture of (poly(3,4-ethylenedioxythiophene) (PEDOT) and poly(styrenesulfonate) (PSS). ${ }^{44,45}$ Aqueous PEDOT:PSS dispersions are commercially available and its properties and uses in device configuration have been widely explored. PEDOT is a polythiophene derivative made up from 3,4-ethylendioxythiophene (EDOT) monomer units and is a positive charge carrying species in the copolymer blend. PSS is a sulfonated polystyrene derivative and is deprotonated to act as a negative counterion and the p-type dopant, stabilizing the charge on the PEDOT (Figure 6). Conductivity in PEDOT is therefore derived from proton conductivity and the movement of a positive hole along the polymer backbone.

PEDOT:PSS has exhibited conductivities of over 3,000 S.cm ${ }^{-1}$ and can be tuned by considering factors such as PEDOT to PSS ratio and the nature of solvent used in thin-film formation. ${ }^{46,47}$ The good solubility of PEDOT:PSS allows for processing using a variety of techniques including spin-coating, ${ }^{48}$ ink-jet printing ${ }^{49}$ and dip-coating. ${ }^{50}$ By aligning the PEDOT:PSS during the film deposition, conductivities can be further improved to $4,600 \mathrm{~S} . \mathrm{cm}^{-1} .^{51}$ Thin-films of PEDOT:PSS have a high transparency in the visible region, are flexible and are stable at high temperatures. ${ }^{52}$ The work of Leo et al. has shown that when replacing ITO with PEDOT:PSS, treated with ethylene glycol, as the transparent electrode material in an organic solar cell conductivities as high as 1418 S.cm ${ }^{-1}$ can be achieved. ${ }^{53}$ These qualities have led to PEDOT:PSS being considered as a replacement for metal oxides in industry, for example in the replacement of indium tin oxide (ITO) as a transparent electrode in optoelectronic devices such as light emitting diodes (LED) and solar cells. ${ }^{54}$

PEDOT was first reported in 1988 in a patent by Jonas and Heywang, ${ }^{55}$ in which they describe the oxidative polymerization of EDOT and low electrical conductivities were reported, without the presence of the PSS counterions. The first example of PSS being used as a counterion was found in 1997 when PEDOT was immersed in a solution of the oxidizing agent iron(III) tris- $p$-toluene sulfonate. ${ }^{56}$ It was determined that using PSS as a counterion led to better surface coverage of the PEDOT as shown by X-ray photoelectron spectroscopy (XPS) spectra of PSS doped PEDOT compared to when doped with to- sylate $\left(\mathrm{TsO}^{-}\right)$anions. Films prepared from an aqueous suspension of PEDOT:PSS will have a low conductivity, generally around $1 \mathrm{~S} . \mathrm{cm}^{-1}$. Enhancement of the conductivity of PEDOT:PSS has been achieved through a collection of techniques known as secondary-doping. ${ }^{52}$ These can involve addition of high-boiling point polar solvents, ionic liquids or surfactants into the aqueous solution. They may also involve treatment of the thin-film with various kinds of organic and inorganic compounds. The first reported attempt of secondary-doping came from Kim et al. ${ }^{57}$ in which the solvents dimethyl sulfoxide (DMSO), $\mathrm{N}, \mathrm{N}$-dimethyl formamide (DMF), tetrahydrofuran (THF) and water were used; an increase from $0.8 \mathrm{Scm}^{-1}$ from the thin-film in water to $80 \mathrm{~S} \mathrm{~cm}^{-1}$ in DMSO was observed. The use of DMSO has been further explored and the effect on conductivity and figure of merit by changing concentrations of the solvent have been investigated. ${ }^{44}$ Conductivities were found to peak at approximately $570 \mathrm{~S} . \mathrm{cm}^{-1}$ with 5 vol. $\%$ of DMSO and a ZT of $9.2 \times 10^{-3}$ was reported.

A conductivity of $3,065 \mathrm{~S} \mathrm{~cm}^{-1}$ has been observed in a $24 \mathrm{~nm}$ thick PEDOT:PSS film when treated with $1 \mathrm{M}$ sulfuric acid $\left(\mathrm{H}_{2} \mathrm{SO}_{4}\right)$ at $160^{\circ} \mathrm{C}^{46}$ The authors attribute this massive increase in conductivity to the partial interchange of PSS counterions with $\mathrm{HSO}_{4}{ }^{-}$anions. When removed the $\mathrm{PSS}^{-}$is protonated to form PSSH. The normal conformation of PEDOT:PSS is coiled, which causes a localization of the charges and is not good for charge mobility however when the counterion is exchanged under treatment of $\mathrm{H}_{2} \mathrm{SO}_{4}$, and $\mathrm{PSS}^{-}$is removed, a more linear polymer backbone conformation is undertaken by the PEDOT which allows for less impeded charge mobility. The use of PEDOT:PSS in energy conversion and storage applications has been too extensive over the years to be covered within the scope of this review, and the reader is referred to reviews specifically covering the many applications of PEDOT:PSS. ${ }^{58-60}$

\section{N-type Organic Thermoelectric Materials}

Even though a TE device consists of two legs; i.e., a p-type and an n-type leg, the research mainly focused on the design and understanding of p-type materials. The heavy focus of research efforts on p-type TE materials has two reasons: (1) The study of n-type organic thermoelectric materials is rather complicated due to the small electron affinities (EAs) $(-3 \mathrm{eV}$ to $-4 \mathrm{eV})$ of n-type materials leading to chemical instabilities. These small EAs facilitate the creation of negatively charged molecules upon reaction with atmospheric water and oxygen. Therefore, molecules possess EAs larger than $\sim 4 \mathrm{eV}$ are desirable for n-type TE materials. (2) The non-availability of high performing n-type organic conductors complicates their study further and most n-type materials developed so far are lacking the performances currently obtained with p-type materials. Nevertheless, several material families are slowly emerging as promising n-type candidates for thermoelectric applications.

Metallo-polymers (Met-Ps), also called organometallic coordination polymers (OMCPs), are polymer structures consisting of metal ions and ligands, where ions act as connectors between the different ligands. The research on thermoelectric properties of Met-Ps is much less developed than for conjugated polymers although they have been widely used for other purposes such as luminescence, non-linear optics, etc. Most of the research efforts on Met-Ps as thermoelectric materials have been conducted by Zhu et al. In 2012, Zhu et al. explored the TE properties of Poly[ $\mathrm{A}_{\mathrm{x}}(\mathrm{M}$-ett) $]$ system, where A being an ion, $\mathrm{M}$ being a metal element and $\mathrm{ett}$ refers to 1,1,2,2-ethenetetrathiolate. ${ }^{64}$ The basic formula of Poly[ $\mathrm{A}_{\mathrm{x}}(\mathrm{M}$-ett)] was first reported by $\mathrm{H}$. Poleschner, but its TE properties have not been investigated until Zhu work. Its amorphous nature for low thermal conductivities and high electrical conductivity $\left(\sigma: 10^{-5} \sim 50{\left.\mathrm{~S} . \mathrm{cm}^{-1}\right)}\right.$ motivated Zhu group to use this Met-P in thermoelectric applications. They synthesized a series of Poly[ $\mathrm{A}_{\mathrm{x}}(M$-ett $\left.)\right] \mathrm{p}$ - and n-type coordination polymers containing ett linking bridge, where $A$ being a counter cation including tetradecyltrimethyl ammonium, tetrabutylammonium, $\mathrm{Na}^{+}, \mathrm{K}^{+}, \mathrm{Ni}^{2+}$, $\mathrm{Cu}^{2+}$ for both $\mathrm{p}$-and n-type materials and $M$ being the coordination metallic ion of $\mathrm{Ni}$ or $\mathrm{Cu}$ (Figure 7). ${ }^{64}$ 
<smiles>C1CC2CCC(C1)S2</smiles>

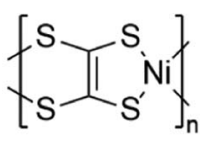

Figure 7. Molecular structures of two coordination polymers, containing $\mathrm{Cu}$ (p-type) or Ni (n-type). The counterions are omitted for clarity.

They have found that materials with $\mathrm{A}=\mathrm{Na}^{+}$and $\mathrm{K}^{+}$and $\mathrm{M}$ $=\mathrm{Ni}^{2+}$ exhibit excellent $\mathrm{n}$-type TE characteristics. The polymer Poly $\left[\mathrm{K}_{\mathrm{x}}(\mathrm{Ni}\right.$-ett $\left.)\right]$ exhibited electrical conductivities up to $63 \mathrm{~S} . \mathrm{cm}^{-1}$ at $440 \mathrm{~K}$, the Seebeck coefficients were in the range of -50 to $-200 \mu \mathrm{VK}^{-1}$. By combining n-type poly[ $\mathrm{Na}_{\mathrm{x}}(\mathrm{Ni}$-ett $\left.)\right]$ and p-type poly $\left[\mathrm{Cu}_{\mathrm{x}}(\mathrm{Cu}\right.$-ett) $]$, they successfully demonstrate the first n-type organic material with an output power of $750 \mu \mathrm{W}$ and a ZT value of $0.1(400 \mathrm{~K})$. One of the drawbacks of these coordination polymers however is their poor solubility in most organic solvents. ${ }^{9}$ Later on, owing to alternative methods in synthetic chemistry smaller and soluble nanoparticles of OMCP have been synthesized. ${ }^{65}$

The research on OMCPs is extended by investigating threedimensional copper 7,7,8,8-tetracyanoquinodimethane ( $\mathrm{Cu}$-TCNQ) coordinated polymers. ${ }^{66}$ The synthesis and TE characterization of CuTCNQ nanocrystals (NC-CuTCNQ) and $\mathrm{Cu}$ - TCNQ nanorod arrays (NrA-CuTCNQ) showed that these materials are strong candidates as n-type TE materials. NC-CuTCNQ deposited my chemical vapor deposition (CVD) on glass exhibits a conductivity of about $0.015 \mathrm{Scm}-1$ at room temperature and $0.037{\mathrm{~S} . \mathrm{cm}^{-1}}^{-1} 370 \mathrm{~K}$ with Seebeck coefficient varies from $-677 \mathrm{mV} . \mathrm{K}^{-1}$ to $-630 \mathrm{mV} . \mathrm{K}^{-1}$ from 290 to $370 \mathrm{~K}$. On the contrary, the TE performance of NrA-CuTCNQ films which was composed of vertically aligned CuTCNQ nanorods was severely restricted. This behavior was attributed to the loose packing of nanorods inhibited the charge transport along the in-plane direction thus lowering electrical conductivity of $0.005 \mathrm{~S} \mathrm{~cm}^{-1}$, three times less than NC-CuTCNQ. Abovementioned encouraging values from metallated polymers opened up a new direction for the development of n-type organic TEs. Another type of thermoelectric device was demonstrated by Crispin et al. by means of ink-jet printing. ${ }^{67}$ The n-type ink component was the crystalline powders of TTF-TCNQ were blended with PVC in toluene. The extrapolated output of the thermoelectric generation consisting of 54 thermo- couples was 0.27 $\mu \mathrm{W} . \mathrm{cm}^{-2}$ at optimum packing density and $\Delta \mathrm{T}=30 \mathrm{~K} .{ }^{67}$

Organic small molecules such as fullerenes and charge transfer complexes are easier to synthesize and crystallize compared to conjugated polymers. Molecular organic semiconductors have attracted great attention in order to meet the requirement of n-type organic TE materials. Fullerenes are one of the best n-type semiconductors with high electron mobility, low thermal conductivity $(\sim 0.4 \mathrm{~W} / \mathrm{mK}),{ }^{68}$ and excellent thermal stability ${ }^{69}$ and it is ideal for many organic electronic
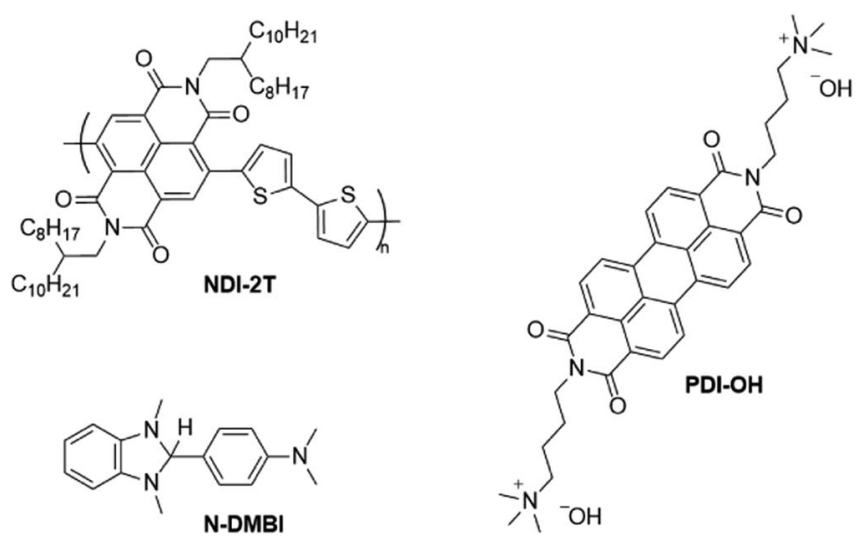

Figure 8. Molecular structures of n-type semiconducting polymer P(NDI2OD-T2), the molecular dopant DMBI and the self-dopable PDI derivative, PDI-OH. applications including organic solar cells and thermoelectric generators (TEGs)..$^{70-72} \mathrm{n}$-doped fullerenes can be achieved by vacuum deposition of several dopants such as salts and alkaline metals. ${ }^{73} \mathrm{TE}$ effect of fullerene $\mathrm{C}_{60}$ was studied using caesium carbonate $\left(\mathrm{Cs}_{2} \mathrm{CO}_{3}\right)$ as n-dopant buffer layer to improve morphology thus higher electron mobility up to $3 \mathrm{~cm}^{2} . \mathrm{V}^{-1} \mathrm{~s}^{-1} .{ }^{74}$ Power factor of $20.5 \mu \mathrm{W} \cdot \mathrm{m}^{-1} \cdot \mathrm{K}^{-2}$ at room temperature was achieved for $\mathrm{Cs}_{2} \mathrm{CO}_{3} / \mathrm{C}_{60}$ bilayer device after optimization. Similar power factors were reported for $\mathrm{C}_{60}$ samples doped with $\mathrm{Cr}_{2}(\mathrm{hpp})_{4}$ or $\mathrm{W}_{2}(\mathrm{hpp})_{4}(\mathrm{hpp}=1,3,4,6,7,8$-hexahydro$2 \mathrm{H}$-pyrimido [1,2-a] pyrimidinato). ${ }^{75} \mathrm{~K}$ and $\mathrm{Rb}$-doped $\mathrm{C}_{60}$ were also used as TE materials and Seebeck coefficients were used to determine the thermopower to be -11 and $-18 \mu \mathrm{V} . \mathrm{K}^{-1}$ at $300 \mathrm{~K} .{ }^{76}$ Several other composites of $\mathrm{C}_{60}{ }^{77}$ have been investigated in terms of their TE properties processed by mechanical alloying and spark plasma sintering. Studies of fullerene derivatives $\mathrm{C}_{60}$ and $\mathrm{C}_{70}$ show comparable power factors to those achieved with charge transfer salts; i.e. TCNQ derivatives. $^{78}$

Charge transfer complexes are interesting materials in TE applications due to their quasi-one-dimensional characters in structures and electronic properties enables intrinsic high electrical conductivities. ${ }^{79}$ Charge transfer co-crystals salts including tetrathiafulvalene (TTF) and tetracyanoquinodimethane (TCNQ) have been attracting interest in TE research as conductivity and thermopower can be tuned along altering crystal directions. ${ }^{80}$ Binder materials such as polyvinylchloride have been blended with charge transfer salts in order to improve their processability and shown to have Seebeck coefficients up to $-48 \mu$ V.K. ${ }^{-1}$ when used as n-type leg in an "all organic" thermoelectric device, where PEDOT:Tos was used as the p-type leg. ${ }^{67}$

As mentioned earlier, a good n-type leg thermoelectric material should possess high electron affinities (EA) for stability. The low EA of organic semiconductors limited the n-tpye leg material options. In order to achieve desirable energetics as well as cost-effective fabrication solution processing methods are required. Another significant point for an efficient n-type TE material is its electrical conductivity. Unlike conventional inorganic semiconductor materials, organic materials can be doped with introduction of large molecular species that may disrupt its molecular organization. Chabinyc and Segalman and others recently demonstrated the introduction of extrinsic dopants that localizes the dopants within the active host matrix and create high electrical conductivity and thermoelectric performance. ${ }^{81-84}$ Recently, perlyenediimides (PDIs) emerged as solution processable organic semiconductors that have high EA $(\sim 4.0 \mathrm{eV})$ where the structural modifications enable to tune the energetic levels. ${ }^{85}$ Chemical modifications of the PDI core can deliver water soluble derivatives enabling a pathway toward non-toxic, solution processing of thin films. PDIs can be self-doped due to tethered tertiary amine functional groups that serve as active sites for low temperature self-doping. ${ }^{81}$ Thus, electrical conductivity $(\sigma)$ and as a result thermoelectric power of PDI molecules can be controlled with side chain engineering. ${ }^{86}$ Efficient n-type doping has been achieved by the addition of pendant trimethyl amine groups to molecular perylene-diimide derivatives, ${ }^{81} \mathrm{Gregg}$ et al. reported a reversible self-doping mechanism of a water soluble PDI derivative through low temperature treatment and conductivities up to $10^{-3}{\mathrm{~S} . \mathrm{cm}^{-1}}$ achieved. ${ }^{84}$ Segalman and co-workers recently presented that self-doped PDIs have one of the highest $n$-type thermoelectric performance of solution-processed organic materials reported so far. ${ }^{82}$

They reported PDI derivatives with various chain lengths that are strongly correlated to the morphology and electronic properties. The study pioneered the work on identification of the active motif responsible for n-type doping in PDIs. The authors demonstrate that hydroxide counterions and the alkyl tethered trimethylammonium functional groups in the PDI system react in the solid-state to produce alkyl tethered dimethylamino end groups are the main precursors in the doping process. Through structural optimization they achieve electrical conductivities and thermoelectric power factors as high as $0.5 \mathrm{~S} . \mathrm{cm}^{-1}$ and $1.4 \mu \mathrm{W} . \mathrm{m}^{-1} \mathrm{~K}^{-2}$, respectively.

A variety of new organometallic complexes, ${ }^{87}$ hydride donors, 1,3Dimethyl-2-phenyl-2,3-dihydro-1H-benzoimidazole (DMBI), ${ }^{88}$ and 
charged tetrabutylammonium salts, in which counter-ions have been proposed to act as sacrificial electron donors are all desirable approaches for extrinsic doping. Recent developments in n-type organic thermoelectric materials suggest that stable extrinsic doping is highly relevant as you can tune the carrier concentration which enables the optimization of thermoelectric figure of merit.

A polymer from naphthalenediimide (NDI) core, $\mathrm{P}(\mathrm{NDI} 2 \mathrm{OD}-\mathrm{T} 2$ ), attracted attention for TE applications due to its bifunctional morphology upon different processing (Figure 8). Low temperature processing $\left(\sim 150^{\circ} \mathrm{C}\right)$ of $\mathrm{P}(\mathrm{NDI} 2 \mathrm{OD}-\mathrm{T} 2)$ result in pi-stacking direction of the polymer chains is perpendicular to the substrate (face-on), whereas high temperature $\left(\sim 300^{\circ} \mathrm{C}\right)$ processing dominates pi-stacking direction and polymer backbone parallel to the substrate (edge-on). These two unique structures result in different morphologies and molecular packing that effect mobilities. ${ }^{89}$ A study of P(NDI2OD-T2) doped with dihydro-1H-benzoimidazol-2-yl (N-DBI) showed that a power factor of $0.6 \mu \mathrm{W} \cdot \mathrm{m}^{-1} \mathrm{~K}^{-2}$ is achievable with a thermopower of -850 $\mu \mathrm{V} . \mathrm{K}^{-1} .{ }^{83,90}$ Pei et al. doped a series of n-type polymers comprising the fused benzodifurandione-based poly(p-phenylene vinylene) (BDPPV) building block with ((4-(1,3-dimethyl-2,3-dihydro-1Hbenzoimidazol-2-yl)phenyl)dimethylamine) N-DMBI in solution..$^{91}$ Selective chemical modifications to the backbone lead to very high conductivities of $14 \mathrm{~S} . \mathrm{cm}^{-1}$ and power factors of $28 \mu \mathrm{W} \cdot \mathrm{m}^{-1} \cdot \mathrm{K}^{-2}$, one of the highest values for solution processable $n$-type thermoelectric materials reported so far.

\section{Graphene and Nanocomposite Materials}

Besides synthesizing bulk materials for TE applications from scratch, blending already known materials to achieve superior thermoelectric properties has been proven to be a successful approach. By blending tellurium nanowires into PEDOT:PSS matrices, the idea is to take advantage of the excellent conducting properties of Te, whilst at the same time keeping the thermal conductivity to a minimum. Several groups have employed the strategy of fabricating tellurium/PEDOT:PSS nanocomposites. ${ }^{92,93}$ Tellurium nanowire/PEDOT: PSS composites have been synthesized and subsequently treated with $\mathrm{H}_{2} \mathrm{SO}_{4}$ to improve the electrical conductivity of the PEDOT: PSS. The optimized power factor achieved was $284 \mu \mathrm{W} \cdot \mathrm{m}^{-1} \cdot \mathrm{K}^{-2}$. Subsequently the electrical conductivity was improved from 11 to $335 \mathrm{~S} . \mathrm{cm}^{-1}$. Despite the lack of an experimentally derived thermal conductivity measurement, a ZT value was estimated to be 0.39 and a demonstrator module was made from the composite, producing $2 \mathrm{mV}$ from human body heat. ${ }^{94}$

The allotropes of carbon, graphene, and carbon nanotubes have intrinsically favorable electronic properties of interest in the fabrication and optimization of thermoelectric, optoelectronic, ${ }^{95}$ piezo electronic ${ }^{96}$ and wearable electronic materials. Several studies involving graphene have obtained data with high electrical conductivity and in some cases the potential to reduce the thermal conductivity by phonon scattering. ${ }^{97-100}$ Pure graphene thermoelectric devices have been fabricated whereby Seebeck coefficient measurements of $90 \mu \mathrm{V} . \mathrm{K}^{-1}$ have been observed by increasing interfacial-layers between 50-1000 layers. ${ }^{101}$ Graphene $(\mathrm{rGO}) /$ fullerene $\left(\mathrm{C}_{60}\right),{ }^{102}(\mathrm{rGO})^{103}$ and, graphene/CNT/nano-composites ${ }^{104,105}$ have been studied by several groups and prepared with PEDOT: PSS. The studies showed an increased power factor in all composites when compared with pure PEDOT: PSS due to an increased electrical conductivity/reduced thermal transport. ${ }^{103}$ Concurrently a synergistic effect between the electronic properties have been shown to enhance ZT value, resulting from phonon scattering and higher Seebeck coefficients. One such study demonstrated that the thermoelectric properties depend on the percentage by weight composition of $\left(\mathrm{C}_{60}\right)$. The optimum power factor $32.4 \mu \mathrm{W} . \mathrm{m}^{-1} . \mathrm{K}^{-2}$ corresponded to the $7: 3$ ratio between $\left(\mathrm{C}_{60}\right)$ and $(\mathrm{rGO})$ respectively. It was found that increasing the percentage composition of (rGO) increased electrical conductivity as well as thermal conductivity; meanwhile $\left(\mathrm{C}_{60}\right)$ increased the Seebeck coefficient while simultaneously limiting the increase in thermal conductivity relative to the increasing electrical conductivity. Due to the increase in thermal conductivity from 0.2 to $2 \mu \mathrm{W} . \mathrm{K}^{-1} \mathrm{~m}^{-1}$ and an optimized Seebeck coefficient of $21.8 \mu \mathrm{V} . \mathrm{K}^{-1}$ the nanohybrid was optimized to give a $\mathrm{ZT}$ value of $0.067 .{ }^{102}$

In other works, graphene polymer composites were fabricated via in-situ polymerization of dispersed graphene in PSS, and subsequent polymerization in the presence of EDOT. The resultant thin films displayed electrical conductivity of $637 \mathrm{~S} \mathrm{~cm}^{-1}$ and a Seebeck coefficient of $26.78 \mu \mathrm{V} . \mathrm{K}^{-1}$ and an optimized power factor of $45 \mu \mathrm{W} . \mathrm{m}^{-1} . \mathrm{K}^{-2}$ which was shown to be $93 \%$ higher than the pristine PEDOT: PSS. ${ }^{103}$ The same research group also investigated hybrid structures of PEDOT: PSS/graphene/MWNT. The composite had superior thermoelectric properties compared to the pristine PEDOT: PSS and PEDOT/graphene and PEDOT/ MWNT composites. The electrical conductivity was $689 \mathrm{~S} \mathrm{~cm}^{-1}$, while the power factor and Seebeck coefficient were $37.08 \mu \mathrm{W} . \mathrm{m}^{-1} . \mathrm{K}^{-2}$ and $23.20 \mu \mathrm{V} . \mathrm{K}^{-1}$ respectively. The ZT value increased from 0.017 for pristine PEDOT: PSS to 0.031 for the PEDOT: PSS/graphene/MWNT composites. ${ }^{104}$ The latest work on these ternary composites reports the effects of different weight ratio between PEDOT/graphene/carbon nanotube based ternary composite as well as the effects of acid treatment on the electrical conductivity and Seebeck coefficient. Electrical conductivities of 208.4 S.cm ${ }^{-1}$ were reported with enhancement to power factors going from 1.96 $\mu \mathrm{W} \cdot \mathrm{m}^{-1} \cdot \mathrm{K}^{-2}$ to $9.10 \mu \mathrm{W} \cdot \mathrm{m}^{-1} \cdot \mathrm{K}^{-2}$ upon acid treatment. ${ }^{105}$ These findings infer the potential for optimizing the thermopower based on synergistic electronic effects between different intrinsic properties presented by the materials selected and device fabrication synthesis methods employed. The PEDOT: PSS acts as a polymer matrix with low thermal conductivity for charge transport ${ }^{106}$ and the CNTs depending on size-diameter may introduce phonon scattering properties, higher Seebeck coefficient and electrical conductivity. ${ }^{107-109}$

Despite having relatively high thermal conductivity, carbon nanotube composites are the focus of much research due to their high electrical conductivity and ease of processing. PEDOT: PSS-carbon nanotubes and polyvinyl acetate composites have been synthesized. ${ }^{110}$ The report showed that thermopower was weakly correlated with the electrical conductivity, resulting in large thermoelectric power factors of $160 \mu \mathrm{W} \cdot \mathrm{m}^{-1} \cdot \mathrm{K}^{-2}$.

The highest room temperature electrical conductivity and Seebeck coefficient along the in-plane direction was shown to be 1350 S.cm ${ }^{-1}$ and $41 \mu \mathrm{V} . \mathrm{K}^{-1}$, respectively. Without additional DMSO doping, PH1000 shows higher thermopowers while PH500 has better electrical conductivities. Carbon nanotube / poly(vinyl chloride) nanocomposites have been fabricated by dispersing carbon nanotubes in an n-type poly(nickel 1,1,2,2-ethenetetra-thiolate) (PETT). ${ }^{21}$ The conductive n-type polymer replaced PEDOT: PSS as a dispersant for CNTs. The optimized power factor for methanol treated PETT-CNTpolyvinyl chloride was $58.6 \mu \mathrm{W} \cdot \mathrm{m}^{-1} \cdot \mathrm{K}^{-2}$, while the electrical conductivity and Seebeck coefficient were, $630 \mathrm{~S} \mathrm{~cm}^{-1}$ and $30.5 \mu \mathrm{V} . \mathrm{K}^{-1}$ respectively and demonstrated a ZT value of $0.3 .^{111}$

Polystyrene has been utilized as a matrix for SWNTs yielding an optimum performance, at $20 \%$ by weight polystyrene, giving a power factor of $85 \mu \mathrm{W} \cdot \mathrm{m}^{-1} \cdot \mathrm{K}^{-2}$, a Seebeck coefficient of $57 \mu \mathrm{V} \cdot \mathrm{K}^{-1}$ and an electrical conductivity of around $285 \mathrm{~S} . \mathrm{cm}^{-1}$. Importantly the thermal conductivity decreases with the addition of polystyrene. The polymer composite offered a 1.7 times higher power factor than the pristine SWNT. ${ }^{112}$ Other work with SWNT shows an improved performance by a layered morphology in the form of SWNTs sandwiched by polypyrrole (PPy) nanowires. The composites show a Seebeck coefficient of $41.3 \mu \mathrm{V} . \mathrm{K}^{-1}$, a conductivity of $802.6 \mathrm{~cm}^{-1}$ and a maximum power factor of $21.7 \mu \mathrm{W} \cdot \mathrm{m}^{-1} \cdot \mathrm{K}^{-2}{ }^{113}$ These results signify the potential for small molecule and polymer thermoelectric device optimization without relying on PEDOT: PSS due to its poor stability and performance in high humidity conditions; as well as the importance of materials interaction within a device.

In addition the studies from Ref. 107 and Ref. 114 shows that the Seebeck coefficient increases in semiconducting-SWNT (S-SWNT) with only a slight increase in thermal conductivity with increasing temperature due to the amorphous grain structure in bulk CNTs. The Studies conducted presented high Seebeck coefficients from different 


\begin{tabular}{|c|c|c|c|c|c|}
\hline Materials type & $\sigma\left(\mathrm{S} . c m^{-1}\right)$ & $S\left(\mu V \cdot K^{-1}\right)$ & $\mathrm{S}^{2} \sigma \mathrm{T} / \lambda\left(\mu \mathrm{W} \cdot \mathrm{m}^{-1} \cdot \mathrm{K}^{-2}\right)$ & $\mathrm{ZT}$ & Ref. \\
\hline Tellurium nanowire-PEDOT: PSS & 334.68 & & 284.00 & 0.390 & 88 \\
\hline \multirow{3}{*}{ graphene } & & 90.00 & & & 95 \\
\hline & & 21.80 & & 0.067 & 96 \\
\hline & 637.00 & 26.78 & 45.00 & & 97 \\
\hline PEDOT/graphene and PEDOT/ MWNT & 689.00 & 23.20 & 37.08 & 0.031 & 98 \\
\hline PEDOT/graphene and PEDOT/ CNTs & 208.40 & & 9.10 & & 99 \\
\hline PEDOT: PSS-graphene- $\mathrm{TiO}_{2}$ & 260.00 & -47.00 & 47.00 & 0.0048 & 121 \\
\hline Carbon nanotubes & 1350.0 & 41.00 & 160.0 & & 104 \\
\hline poly(vinyl chloride)-CNTs & 629.90 & 30.50 & 58.60 & 0.30 & 105 \\
\hline Polystyrene-SWNT & 285.00 & 57.00 & 85.00 & & 106 \\
\hline polypyrrole (PPy)-SWNT & 802.60 & 41.30 & 21.70 & & 107 \\
\hline Pure S-SWNT & & 170.0 & & & 101 \\
\hline Acid treated-S-SWNT & & & 108.6 & 0.15 & 101 \\
\hline perovskites (dark) & & 820.0 & & & 117 \\
\hline Perovskites (illumination) & & 540.0 & & & 117 \\
\hline Lathanun oxide perovskites & & -200 & & & 120 \\
\hline Doped lanthanum perovskites & & 450 & & & 120 \\
\hline
\end{tabular}

methods including treatment of metallic and S-SWNT with different acids. It appears from several studies ${ }^{115,116}$ that by filtering out metallic CNTs high Seebeck coefficients may be induced by the S-SWNTs. Other articles describe the research potentials in filtering the different forms of nanotubes and implications of the diameter and size of the nanotubes in relations to thermal conductance, electrical conductance and optoelectronic properties. ${ }^{117,118}$ One particular study focusing on semi-conducting SWCNT boasts a high Seebeck coefficient of 170 $\mu \mathrm{V} . \mathrm{K}^{-1}$ presented by the un-doped S-SWNT but with a much lower power factor owning to the low conductivity of the S-SWNT. On the other hand the acid treatment may have decreased resistivity however the Seebeck decreased also giving an estimated ZT of 0.15 and power factor of $109 \mu \mathrm{W} \cdot \mathrm{m}^{-1} \cdot \mathrm{K}^{-2}$ at $300 \mathrm{~K} .^{107}$

Lead halide perovskites have recently become a hot topic in the area of photovoltaics ${ }^{119-122}$ and indeed it has been shown to have the potential for high Seebeck as high as $\left(\sim 10^{5} \mu \mathrm{V} . \mathrm{K}^{-1}\right)$ at room temperature. ${ }^{123} \mathrm{Ab}$ initio calculations suggest $\mathrm{ZT}$ values of $1-2$ for $\mathrm{CH}_{3} \mathrm{NH}_{3} \mathrm{PbI}_{3}$ and $\mathrm{CH}_{3} \mathrm{NH}_{3} \mathrm{SnI}_{3}$ perovskites, due to high carrier mobility, small carrier effective masses and weak electron-phonon and hole phonon couplings. ${ }^{124}$ other studies experimentally determine high Seebeck coefficients of $820 \mu \mathrm{V} \cdot \mathrm{K}^{-1}$ (measured in the dark) to $540 \mu \mathrm{V} . \mathrm{K}^{-1}$ (measured under illumination). ${ }^{125}$ However due to the low electrical conductivity $0.05 \mathrm{~S} \mathrm{~cm}^{-1}$ owning to a low carrier concentration doping is necessary in order to increase conductance. ${ }^{126}$ Interestingly the perovskite material exhibits different Seebeck coefficients depending on light environment due to charge availability upon photon excitation. ${ }^{125}$ The same study conducted several experiments with doped $\mathrm{CH}_{3} \mathrm{NH}_{3} \mathrm{SnI}_{3}$ showing the potential for high $\mathrm{ZT}$ of 3 with the optimum electrical conductivity.

Organic-inorganic lead halide perovskites with $\mathrm{ABX}_{3}$ structure are found to be intrinsic semiconductor materials ${ }^{127}$ and so doping may be required to impart p-type or n-type behavior. Replacement of the one or more of the $\mathrm{A}$ and $\mathrm{B}$ cations, or the $\mathrm{X}$ anion has resulted in bandgap tuning and stability improvements. ${ }^{128}$ Doping by replacement of the A cation with can present problems due to spatial restrictions inside the perovskite crystal lattice where the A cation sits inside the three-dimensional $\mathrm{BX}_{6}$ octahedral. ${ }^{129}$ Most doping studies are carried out with homovalent ions, which do not change the sign or concentration of the majority charge carriers. Doping using trivalent cations such as $\mathrm{Bi}^{3+}$ has been achieved but doping organic-inorganic lead halide perovskites presents a challenge since differently charged cations should satisfy both octahedral and tolerance factors while maintaining the same underlying crystal structure. ${ }^{130}$

Other lanthanum cobalt oxide and titanium based type perovskites have been synthesized and high Seebeck coefficient of $-200 \mu \mathrm{V} . \mathrm{K}^{-1}$ have been obtained by controlling the spin state of the cations and a sign change from positive to negative with $\mathrm{Ti}^{4+}$ doping. ${ }^{131}$ Higher Seebeck coefficients are reported in the article ranging from -200 to $450 \mu \mathrm{V} \cdot \mathrm{K}^{-1}$ depending on the doping type and concentrations. Research into increasing the electrical conductivity of these materials without adversely affecting their low thermal conductance would prove beneficial as potential candidates in both thermoelectric, ${ }^{132}$ and thermo-photovoltaics devices. ${ }^{133}$

\section{Conclusions}

The figure of merit of n-type materials for thermoelectric generators is certainly a factor that needs consideration. In addition to self-doping, the improvement in methods that can stably n-dope the organic materials will continue to attract attention in TE research. Despite substantial growth in n-type material research, there are still limitations in order to develop n-type conductors matching the carrier mobilities to those of p-type materials. Instead of relying on organic semiconductors and dopants developed for other applications such as organic field effect transistors and solar cells, it is important to design and synthesize organic n-type conductors specifically for thermoelectric applications, thereby specifically focusing on solution processability, electron mobility, packing and doping efficiency. The development in solution processable n-type semiconductors will not only contribute to maximizing the power factor in organic thermoelectrics, but also to the field of organic electronics in general by providing a compelling doping platform to reduce injection barriers and ohmic losses in organic photovoltaic cells (OPV), light emitting diodes (OLED) and field effect transistors (OFET).

The development of thermoelectric module technology using printing methods has the potential to be considered for waste management products. The development of patterning techniques may facilitate the complexity of printing $\mathrm{p}$ - and n-type legs. With the growing attention to solution processable organic thermoelectric materials, it is possible to imagine using high throughput techniques for the large area applications in the future. All-organic thermoelectric devices have been demonstrated utilizing screen-printing and R2R printing. The low temperature applicability of solution processed semiconductors makes TE materials more appealing to be potential candidates for wearable electronics.

\section{Acknowledgments}

L.M.C and B.C.S. acknowledge the Materials Research Institute and Queen Mary University of London for financial support. M.J.C. would like to thank the EPSRC funded (EP/N506553/1) College of Engineering Doctoral Training Partnership at Swansea University.

\section{References}

1. C. Forman, I. K. Muritala, R. Pardemann, and B. Meyer, "Estimating the global waste heat potential," Renewable and Sustainable Energy Reviews, 57, 1568 (2016). 
2. J. M. Cullen and J. M. Allwood, "Theoretical efficiency limits for energy conversion devices," Energy, 35, 2059 (2010)

3. L. E. Bell, "Cooling, Heating, Generating Power, and Recovering Waste Heat with Thermoelectric Systems," Science, 321, 1457 (2008).

4. F. J. DiSalvo, "Thermoelectric Cooling and Power Generation," Science, 285, 703 (1999).

5. H. Hubert Peltier Effect Heat Pump. USA patent 3,635,037 (1972).

6. O. Bubnova and X. Crispin, "Towards polymer-based organic thermoelectric generators," Energy \& Environmental Science, 5, 9345 (2012).

7. H. E. Katz and T. O. Poehler Innovative Thermoelectric Materials: Polymer, Nanostructure and Composite Thermoelectrics. 11 (Imperial College Press, 2016)

8. S. LeBlanc, "Thermoelectric generators: Linking material properties and systems engineering for waste heat recovery applications," Sustainable Materials and Technologies, 1-2, 26 (2014)

9. Q. Zhang, Y. Sun, W. Xu, and D. Zhu, "Organic Thermoelectric Materials: Emerging Green Energy Materials Converting Heat to Electricity Directly and Efficiently," Advanced Materials, 26, 6829 (2014).

10. M. S. Freund and D. Bhavana Self-doped Conducting Polymers. 1 edn, 1 (John Wiley \& Sons, 2007).

11. A. R. West Solid State Chemistry and it's Applications. 497 (John Wiley \& Sons, 1987).

12. B. Lüssem, M. Riede, and K. Leo, "Doping of organic semiconductors," physica status solidi (a), 210, 9 (2013).

13. H. Méndez et al., "Doping of Organic Semiconductors: Impact of Dopant Strength and Electronic Coupling," Angewandte Chemie International Edition, 52, 775 (2013).

14. I. Salzmann, G. Heimel, M. Oehzelt, S. Winkler, and N. Koch, "Molecular Electrical Doping of Organic Semiconductors: Fundamental Mechanisms and Emerging Dopant Design Rules," Accounts of Chemical Research, 49, 370 (2016).

15. C. K. Chiang et al., "Electrical Conductivity in Doped Polyacetylene," Physical Review Letters, 39, 1098 (1977)

16. H. Shirakawa, E. J. Louis, A. G. MacDiarmid, C. K. Chiang, and A. J. Heeger, "Synthesis of electrically conducting organic polymers: halogen derivatives of polyacetylene, (CH)," Journal of the Chemical Society, Chemical Communications, 578 (1977).

17. E. Poverenov, N. Zamoshchik, A. Patra, Y. Ridelman, and M. Bendikov, "Unusual Doping of Donor-Acceptor-Type Conjugated Polymers Using Lewis Acids," Journal of the American Chemical Society, 136, 5138 (2014).

18. P. Pingel et al., "p-Type Doping of Poly(3-hexylthiophene) with the Strong Lewis Acid Tris(pentafluorophenyl)borane," Advanced Electronic Materials, 2, 1600204 n/a (2016).

19. G. Parthasarathy, C. Shen, A. Kahn, and S. R. Forrest, "Lithium doping of semiconducting organic charge transport materials," Journal of Applied Physics, 89, 4986 (2001).

20. L. S. Hung, C. W. Tang, and M. G. Mason, "Enhanced electron injection in organic electroluminescence devices using an $\mathrm{Al} / \mathrm{LiF}$ electrode," Applied Physics Letters, 70, 152 (1997).

21. M. G. Mason et al., "Interfacial chemistry of Alq3 and LiF with reactive metals," Journal of Applied Physics, 89, 2756 (2001)

22. T. Senku et al., "Doping Effect of Tetrathianaphthacene Molecule in Organic Semiconductors on Their Interfacial Electronic Structures Studied by UV Photoemission Spectroscopy," Japanese Journal of Applied Physics, 44, 3760 (2005).

23. J. L. Bredas and G. B. Street, "Polarons, bipolarons, and solitons in conducting polymers," Accounts of Chemical Research, 18, 309 (1985)

24. W. P. Su, J. R. Schrieffer, and A. J. Heeger, "Solitons in Polyacetylene," Physical Review Letters, 42, 1698 (1979).

25. W. P. Su, J. R. Schrieffer, and A. J. Heeger, "Soliton excitations in polyacetylene," Physical Review B, 22, 2099 (1980).

26. W. P. Su and J. R. Schrieffer, "Soliton Dynamics in Polyacetylene," Proceedings of the National Academy of Sciences of the United States of America, 77, 5626 (1980).

27. Ali Benamara A., M. Galtier, and A. Montaner, "N doping of polyacetylene," Synthetic Metals, 41, 45 (1991).

28. R. Zuzok, A. B. Kaiser, W. Pukacki, and S. Roth, "Thermoelectric power and conductivity of iodine-doped "new" polyacetylene," The Journal of Chemical Physics, 95, 1270 (1991)

29. J. Płocharski, W. Pukacki, and S. Roth, "Electrical conductivity of iodine doped oriented polyacetylene," Synthetic Metals, 41, 133 (1991).

30. S. P. Armes, "Optimum reaction conditions for the polymerization of pyrrole by iron(III) chloride in aqueous solution," Synthetic Metals, 20, 365 (1987).

31. D. Oukil et al., "Electrochemical synthesis of polypyrrole films doped by ferrocyanide ions onto iron substrate: Application in the electroanalytical determination of uric acid," Sensors and Actuators B: Chemical, 204, 203 (2014).

32. D. D. Ateh, H. A. Navsaria, and P. Vadgama, "Polypyrrole-based conducting polymers and interactions with biological tissues," Journal of the Royal Society Interface, 3, 741 (2006)

33. G. Qi, L. Huang, and H. Wang, "Highly conductive free standing polypyrrole films prepared by freezing interfacial polymerization," Chemical Communications, $\mathbf{4 8 ,}$ 8246 (2012)

34. N. Kemp et al., "Thermoelectric power and conductivity of different types of polypyrrole," Journal of Polymer Science Part B: Polymer Physics, 37, 953 (1999).

35. S. Bhadra, N. K. Singha, and D. Khastgir, "Electrochemical synthesis of polyaniline and its comparison with chemically synthesized polyaniline," Journal of Applied Polymer Science, 104, 1900 (2007).

36. I. Y. Choi et al., "High-Conductivity Two-Dimensional Polyaniline Nanosheets Developed on Ice Surfaces," Angewandte Chemie International Edition, 54, 10497 (2015).
37. J. Stejskal et al., "Polyaniline prepared in the presence of various acids: a conductivity study," Polymer International, 53, 294 (2004).

38. Y. Yang, S. Chen, and L. Xu, "Enhanced Conductivity of Polyaniline by Conjugated Crosslinking," Macromolecular Rapid Communications, 32, 593 (2011).

39. J. Li, X. Tang, H. Li, Y. Yan, and Q. Zhang, "Synthesis and thermoelectric properties of hydrochloric acid-doped polyaniline," Synthetic Metals, 160, 1153 (2010).

40. A. Marrocchi, D. Lanari, A. Facchetti, and L. Vaccaro, "Poly(3-hexylthiophene): synthetic methodologies and properties in bulk heterojunction solar cells," Energy \& Environmental Science, 5, 8457 (2012)

41. M. J. Panzer and C. D. Frisbie, "High Carrier Density and Metallic Conductivity in Poly(3-hexylthiophene) Achieved by Electrostatic Charge Injection," Advanced Functional Materials, 16, 1051 (2006).

42. Y. Xuan et al., "Thermoelectric properties of conducting polymers: The case of poly(3-hexylthiophene)," Physical Review B, 82, 115454 (2010).

43. Q. Zhang, Y. Sun, W. Xu, and D. Zhu, "Thermoelectric energy from flexible P3HT films doped with a ferric salt of triflimide anions," Energy \& Environmental Science, 5, 9639 (2012)

44. M. Scholdt et al., "Organic Semiconductors for Thermoelectric Applications," Journal of Electronic Materials, 39, 1589 (2010).

45. L. Groenendaal, F. Jonas, D. Freitag, H. Pielartzik, and J. R. Reynolds, "Poly(3,4 ethylenedioxythiophene) and Its Derivatives: Past, Present, and Future," Advanced Materials, 12, 481 (2000)

46. Y. Lin, P. Cheng, Y. Li, and X. Zhan, "A 3D star-shaped non-fullerene acceptor for solution-processed organic solar cells with a high open-circuit voltage of $1.18 \mathrm{~V}$," Chemical Communications, 48, 4773 (2012).

47. S. K. M. Jönsson et al., "The effects of solvents on the morphology and sheet resistance in poly(3,4-ethylenedioxythiophene)-polystyrenesulfonic acid (PEDOTPSS) films," Synthetic Metals, 139, 1 (2003).

48. T.-C. Li and R.-C. Chang, "Improving the performance of ITO thin films by coating PEDOT:PSS," International Journal of Precision Engineering and ManufacturingGreen Technology, 1, 329 (2014)

49. F. Ely et al., "Patterning quality control of inkjet printed PEDOT:PSS films by wetting properties," Synthetic Metals, 161, 2129 (2011).

50. L. Huang, Z. Hu, K. Zhang, P. Chen, and Y. Zhu, "Dip-coating of poly $(3,4$ ethylenedioxythiophene):poly(styrenesulfonate) anodes for efficient polymer solar cells," Thin Solid Films, 578, 161 (2015).

51. B. J. Worfolk et al., "Ultrahigh electrical conductivity in solution-sheared polymeric transparent films," Proceedings of the National Academy of Sciences, 112, 14138 (2015).

52. J. Ouyang, "'Secondary doping" methods to significantly enhance the conductivity of PEDOT:PSS for its application as transparent electrode of optoelectronic devices," Displays, 34, 423 (2013)

53. Y. H. Kim et al., "Highly Conductive PEDOT:PSS Electrode with Optimized Solvent and Thermal Post-Treatment for ITO-Free Organic Solar Cells," Advanced Functional Materials, 21, 1076 (2011)

54. Z. Yu, Y. Xia, D. Du, and J. Ouyang, "PEDOT:PSS Films with Metallic Conductivity through a Treatment with Common Organic Solutions of Organic Salts and Their Application as a Transparent Electrode of Polymer Solar Cells," ACS Applied Materials \& Interfaces, 8, 11629 (2016).

55. F. D. C. D. Jonas, G. D. C. D. Heywang, and W. Schmidtberg (Google Patents, 1989)

56. K. Z. Xing, M. Fahlman, X. W. Chen, O. Inganäs, and W. R. Salaneck, "The electronic structure of poly(3,4-ethylene-dioxythiophene): studied by XPS and UPS,' Synthetic Metals, 89, 161 (1997).

57. J. Y. Kim, J. H. Jung, D. E. Lee, and J. Joo, "Enhancement of electrical conductivity of poly(3,4-ethylenedioxythiophene)/poly(4-styrenesulfonate) by a change of solvents," Synthetic Metals, 126, 311 (2002).

58. R. Yue and J. Xu, "Poly(3,4-ethylenedioxythiophene) as promising organic thermoelectric materials: A mini-review," Synthetic Metals, 162, 912 (2012).

59. K. Sun et al., "Review on application of PEDOTs and PEDOT:PSS in energy conversion and storage devices," Journal of Materials Science: Materials in Electronics, 26, 4438 (2015).

60. H. Shi, C. Liu, Q Jiang, and J. Xu "Effective Approaches to Improve the Electrical Conductivity of PEDOT:PSS: A Review," Advanced Electronic Materials, 1 1500017-n/a (2015)

61. D. Venkateshvaran et al., "Approaching disorder-free transport in high-mobility conjugated polymers,"Nature, 515, 384 (2014).

62. D. Venkateshvaran, A. J. Kronemeijer, J. Moriarty, D. Emin, and H. Sirringhaus, "Field-effect modulated Seebeck coefficient measurements in an organic polyme using a microfabricated on-chip architecture," APL Materials, 2, 032102 (2014).

63. F. Zhang et al., "Modulated Thermoelectric Properties of Organic Semiconductors Using Field-Effect Transistors," Advanced Functional Materials, 25, 3004 (2015).

64. Y. Sun et al., "Organic Thermoelectric Materials and Devices Based on p- and n-Type Poly(metal 1,1,2,2-ethenetetrathiolate)s," Advanced Materials, 24, 932 (2012).

65. C. Faulmann et al., "Nickel ethylene tetrathiolate polymers as nanoparticles: a new synthesis for future applications?," Journal of Nanoparticle Research, 15, 1586 (2013).

66. Y. Sun et al., "n-Type thermoelectric materials based on CuTCNQ nanocrystals and CuTCNQ nanorod arrays," Journal of Materials Chemistry A, 3, 2677 (2015).

67. O. Bubnova et al., "Optimization of the thermoelectric figure of merit in the conducting polymer poly(3,4-ethylenedioxythiophene)," Nat Mater, 10, 429 (2011).

68. N. H. Tea et al., "Thermal conductivity of C60 and C70 crystals," Applied Physics A, 56, 219 (1993).

69. A. M. Vassallo, L. S. K. Pang, P. A. Cole-Clarke, and M. A. Wilson, "Emission FTIR study of C60 thermal stability and oxidation," Journal of the American Chemical Society, 113, 7820 (1991). 
70. D. Baran et al., "Spectroelectrochemical and Photovoltaic Characterization of a Solution-Processable n-and-p Type Dopable Pyrrole-Bearing Conjugated Polymer," Macromolecular Chemistry and Physics, 211, 2602 (2010).

71. S. Holliday et al., "High-efficiency and air-stable P3HT-based polymer solar cells with a new non-fullerene acceptor," Nature Communications, 7, 11585 (2016).

72. D. Baran et al., "Qualitative Analysis of Bulk-Heterojunction Solar Cells withou Device Fabrication: An Elegant and Contactless Method," Journal of the American Chemical Society, 136, 10949 (2014).

73. R. C. Haddon et al., "Conducting films of C60 and C70 by alkali-metal doping," Nature, 350, 320 (1991).

74. M. Sumino et al., "Thermoelectric properties of n-type C60 thin films and their application in organic thermovoltaic devices," Applied Physics Letters, 99, 093308 (2011).

75. Y. Qi et al., "Solution doping of organic semiconductors using air-stable n-dopants," Applied Physics Letters, 100, 083305 (2012).

76. T. Inabe et al., "Electronic structure of alkali metal doped $\mathrm{C}_{60}$ derived from thermoelectric power measurements," Physical Review Letters, 69, 3797 (1992).

77. M. Popov et al., "C60-doping of nanostructured Bi-Sb-Te thermoelectrics," physica status solidi (a), 208, 2783 (2011)

78. T. Menke, D. Ray, J. Meiss, K. Leo, and M. Riede, "In-situ conductivity and Seebeck measurements of highly efficient n-dopants in fullerene C60," Applied Physics Letters, 100, 093304 (2012)

79. M. Hiraoka et al., "Ink-jet printing of organic metal electrodes using charge-transfer compounds," Applied Physics Letters, 89, 173504 (2006).

80. R. Schlitz, A. Glaudell, and M. Chabinyc in Innovative Thermoelectric Materials 39 (IMPERIAL COLLEGE PRESS, 2016).

81. B. Russ et al., "Tethered tertiary amines as solid-state n-type dopants for solutionprocessable organic semiconductors," Chemical Science, 7, 1914 (2016).

82. B. Russ et al., "Power Factor Enhancement in Solution-Processed Organic nType Thermoelectrics Through Molecular Design," Advanced Materials, 26, 3473 (2014).

83. R. A. Schlitz et al., "Solubility-Limited Extrinsic n-Type Doping of a High Electron Mobility Polymer for Thermoelectric Applications," Advanced Materials, 26, 2825 (2014).

84. T. H. Reilly, A. W. Hains, H.-Y. Chen, and B. A. Gregg, "A Self-Doping, O2-Stable, n-Type Interfacial Layer for Organic Electronics," Advanced Energy Materials, 2, 455 (2012).

85. X. Zhan et al., "Rylene and Related Diimides for Organic Electronics," Advanced Materials, 23, 268 (2011).

86. B. Russ, A. Glaudell, J. J. Urban, M. L. Chabinyc, and R. A. Segalman, "Organic thermoelectric materials for energy harvesting and temperature control," Nature Reviews Materials, 1, 16050 (2016).

87. S. Guo et al., "n-Doping of Organic Electronic Materials using Air-Stable Organometallics," Advanced Materials, 24, 699 (2012).

88. B. D. Naab et al., "Mechanistic Study on the Solution-Phase n-Doping of 1,3-Dimethyl-2-aryl-2,3-dihydro-1H-benzoimidazole Derivatives," Journal of the American Chemical Society, 135, 15018 (2013).

89. S. J. Brown, R. A. Schlitz, M. L. Chabinyc, and J. A. Schuller, "Morphologydependent optical anisotropies in the $\exists$ n $\exists$-type polymer P(NDI2OD-T2)," Physical Review B, 94, 165105 (2016).

90. S. N. Patel and M. L. Chabinyc, "Anisotropies and the thermoelectric properties of semiconducting polymers," Journal of Applied Polymer Science, 134, n/a-n/a (2017).

91. K. Shi et al., "Toward High Performance n-Type Thermoelectric Materials by Rational Modification of BDPPV Backbones," Journal of the American Chemical Society, 137, 6979 (2015).

92. K. C. See et al., "Water-processable polymer-nanocrystal hybrids for thermoelectrics," Nano Letters, 10, 4664 (2010).

93. S. K. Yee, N. E. Coates, A. Majumdar, J. J. Urban, and R. A. Segalman, "Thermoelectric power factor optimization in PEDOT:PSS tellurium nanowire hybrid composites," Physical Chemistry Chemical Physics, 15, 4024 (2013).

94. E. Jin Bae, Y. Hun Kang, Jang K.-S. , and S. Yun Cho, "Enhancement of Thermoelectric Properties of PEDOT:PSS and Tellurium-PEDOT:PSS Hybrid Composites by Simple Chemical Treatment," Scientific reports, 6, 18805 (2016).

95. M. Acik and S. B. Darling, "Graphene in perovskite solar cells: device design, characterization and implementation," Journal of Materials Chemistry A, 4, 6185 (2016).

96. A. Sanli, C. Müller, O. Kanoun, C. Elibol, and M. F. X. Wagner, "Piezoresistive characterization of multi-walled carbon nanotube-epoxy based flexible strain sensitive films by impedance spectroscopy," Composites Science and Technology, $\mathbf{1 2 2}$ 18 (2016).

97. K. Yong-Jin, K. Yuna, N. Konstantin, and H. Byung Hee, "Engineering electrical properties of graphene: chemical approaches," 2D Materials, 2, 042001 (2015).

98. A. Y. Serov, Z.-Y. Ong, and E. Pop, "Effect of grain boundaries on thermal transport in graphene," Applied Physics Letters, 102, 033104 (2013).

99. Y. Wang, Z. Song, and Z. Xu, "Characterizing phonon thermal conduction in polycrystalline graphene," Journal of Materials Research, 29, 362 (2014).

100. H. K. Liu, Y. Lin, and S. N. Luo, "Grain Boundary Energy and Grain Size Dependences of Thermal Conductivity of Polycrystalline Graphene," The Journal of Physical Chemistry C, 118, 24797 (2014).

101. L. Mahmoud et al., "Characterization of a Graphene-Based Thermoelectric Generator Using a Cost-Effective Fabrication Process," Energy Procedia, 75, 615 (2015).

102. K. Zhang, Y. Zhang, and S. Wang, "Enhancing thermoelectric properties of organic composites through hierarchical nanostructures," Sci. Rep., 3, 3447 (2013).

103. D. Yoo, J. H. J. J. H. J. J. H. Kim, and J. H. J. J. H. J. J. H. Kim, "Direct synthesis of highly conductive poly(3,4-ethylenedioxythiophene):Poly(4-styrenesulfonate) (PE-
DOT:PSS)/graphene composites and their applications in energy harvesting systems," Nano Research, 7, 717 (2014).

104. D. Yoo et al., "hybridization of PEDOT : PSS on the power factor of polymer thermoelectric energy conversion," Journal of Materials Chemistry A: Materials for energy and sustainability, 3, 6526 (2015).

105. X. Li, L. Liang, M. Yang, G. Chen, and C.-Y. Guo, "Poly(3,4ethylenedioxythiophene)/graphene/carbon nanotube ternary composites with improved thermoelectric performance," Organic Electronics, 38, 200 (2016).

106. Q. Wei, M. Mukaida, K. Kirihara, Y. Naitoh, and T. Ishida, "Recent Progress on PEDOT-Based Thermoelectric Materials," Materials, 8, 732 (2015).

107. N. Yusuke et al., "Giant Seebeck coefficient in semiconducting single-wall carbon nanotube film," Applied Physics Express, 7, 025103 (2014).

108. B. Xu, J. Yin, and Z. Liu in Physical and Chemical Properties of Carbon Nanotubes Ch. 0 (InTech, 2013).

109. A. Benchirouf et al., "Electrical properties of multi-walled carbon nanotubes/PEDOT:PSS nanocomposites thin films under temperature and humidity effects," Sensors and Actuators B: Chemical, 224, 344 (2016).

110. C. Yu, K. Choi, L. Yin, and J. C. Grunlan, "Correction to Light-Weight Flexible Carbon Nanotube Based Organic Composites with Large Thermoelectric Power Factors," ACS Nano, 7, 9506 (2013).

111. N. Toshima et al., "Novel hybrid organic thermoelectric materials:three-component hybrid films consisting of a nanoparticle polymer complex, carbon nanotubes, and vinyl polymer," Advanced materials (Deerfield Beach, Fla.), 27, 2246 (2015).

112. K. Suemori, Y. Watanabe, and S. Hoshino, "Increase in thermoelectric power factor of carbon-nanotube films after addition of polystyrene," Organic Electronics: physics, materials, applications, 28, 135, 2015 (2016)

113. L. Liang, G. Chen, and C. Y. Y. Guo, "Enhanced thermoelectric performance by self-assembled layered morphology of polypyrrole nanowire/single-walled carbon nanotube composites," Composites Science and Technology, 129, 130 (2016)

114. H.-L. Zhang et al., "Electrical and thermal properties of carbon nanotube bulk materials: Experimental studies for the $\$ 328 \backslash$ char 21\{\} $958 \backslash$ phantom $\{\backslash$ rule $\{0.3 \mathrm{em}\}\{0 \mathrm{ex}\}\} \backslash \operatorname{mathrm}\{\mathrm{K}\} \$ \quad$ temperature range," Physical Review B, 75, 205407 (2007).

115. J. P. Small, K. M. Perez, and P. Kim, "Modulation of Thermoelectric Power of Individual Carbon Nanotubes," Physical Review Letters, 91, 256801 (2003).

116. M. S. Arnold, A. A. Green, J. F. Hulvat, S. I. Stupp, and M. C. Hersam, "Sorting carbon nanotubes by electronic structure using density differentiation," Nat Nano, 1, 60 (2006)

117. Y. Kazuhiro, M. Yasumitsu, and K. Hiromichi, "Optical and Conductive Characteristics of Metallic Single-Wall Carbon Nanotubes with Three Basic Colors; Cyan, Magenta, and Yellow," Applied Physics Express, 1, 034003 (2008).

118. S. Kuwahara, Y. Kuwahara, and H. Shinohara, "Quantitative Analysis of Isolated Single-Wall Carbon Nanotubes with Their Molar Absorbance Coefficients," Journal of Nanomaterials, 2014, 6 (2014).

119. M. M. Lee, J. Teuscher, T. Miyasaka, T. N. Murakami, and H. J. Snaith, "Efficient hybrid solar cells based on meso-superstructured organometal halide perovskites," Science (New York, N.Y.), 338, 643 (2012).

120. H.-S. Kim et al., "Lead Iodide Perovskite Sensitized All-Solid-State Submicron Thin Film Mesoscopic Solar Cell with Efficiency Exceeding 9\{\%\}," Scientific, 2, 1 (2012).

121. M. J. Carnie et al., "A one-step low temperature processing route for organolead halide perovskite solar cells," Chem. Commun., 49, 7893 (2013).

122. I. Meager et al., "Thieno[3,2-b]thiophene Flanked Isoindigo Polymers for High Performance Ambipolar OFET Applications," Advanced Functional Materials, 24, 7109 (2014)

123. C. C. Stoumpos, C. D. Malliakas, and M. G. Kanatzidis, "Semiconducting tin and lead iodide perovskites with organic cations: Phase transitions, high mobilities, and near-infrared photoluminescent properties," Inorganic Chemistry, 52, 9019 (2013).

124. Y. He and G. Galli, "Perovskites for Solar Thermoelectric Applications: A First Principle Study of CH 3 NH 3 AI 3 (A = Pb and Sn)," Chemistry of Materials, 26, 5394 (2014)

125. X. Mettan et al., "Tuning of the Thermoelectric Figure of Merit of CH3NH3MI3 $(\mathrm{M}=\mathrm{Pb}, \mathrm{Sn})$ Photovoltaic Perovskites," The Journal of Physical Chemistry C, 119, 11506 (2015).

126. F. Hao, C. C. C. Stoumpos, D. H. H. Cao, R. P. H. P. H. Chang, and M. G. G. Kanatzidis, "Lead-free solid-state organic-inorganic halide perovskite solar cells," Nature Photonics, 8, 489 (2014).

127. K. Miyano, N. Tripathi, M. Yanagida, and Y. Shirai, "Lead Halide Perovskite Photovoltaic as a Model p-i-n Diode," Accounts of Chemical Research, 49, 303 (2016).

128. N. Arora et al., "Intrinsic and Extrinsic Stability of Formamidinium Lead Bromide Perovskite Solar Cells Yielding High Photovoltage," Nano Letters, 16, 7155 (2016).

129. A. M. Ganose, C. N. Savory, and D. O. Scanlon, "Beyond methylammonium lead iodide: prospects for the emergent field of ns 2 containing solar absorbers," Chemical Communications, 53, 20 (2017).

130. A. L. Abdelhady et al., "Heterovalent Dopant Incorporation for Bandgap and Type Engineering of Perovskite Crystals," The Journal of Physical Chemistry Letters, 7, 295 (2016)

131. S. Hébert et al., "Thermoelectric properties of perovskites: Sign change of the Seebeck coefficient and high temperature properties," Progress in Solid State Chemistry, 35, 457 (2007).

132. A. Dey et al., "Polymer based graphene/titanium dioxide nanocomposite (GTNC): an emerging and efficient thermoelectric material," Dalton Transactions, 44, 19248 (2015).

133. W. G. J. H. M. v. Sark, "Feasibility of photovoltaic - Thermoelectric hybrid modules," Applied Energy, 88, 2785 (2011). 